\title{
Rheology properties of carbon nanotube thick film paste for potential application in patch antenna
}

\begin{abstract}
This work investigated the effect of various carbon nanotube (CNT) powders' weight ratio for the preparation of CNT thick film paste. CNT pastes have been prepared with powder weight ratios of $5 \mathrm{wt} \%, 10 \mathrm{wt} \%, 15 \mathrm{wt} \%$ and $20 \mathrm{wt} \%$ to study the rheological properties of the paste. Results show that pastes with a weight ratio of $15 \mathrm{wt} \%$ and $20 \mathrm{wt} \%$ have high viscosity at a low shear rate and decreasing viscosity as shear rates increases. This shear-thinning rheological behaviour is found to be suitable for screen printing pastes. Measurement results of CNT thick film patch antenna show that CNT thick film significantly widens the bandwidth of the antenna while having good return loss. The frequency shift as compared with an antenna with the silver conductive film however notably increases, indicating that electrical properties of CNT patch are lower than those of silver patch, which may occur due to the defects of CNTs in the paste.
\end{abstract}

Keyword: Carbon nanotubes; Thick film; Patch antenna 